\title{
Construction of a Massive Heterogeneous Minority Cultural Resource Integration Model based on Ontology
}

\author{
Ying Liu ${ }^{\mathrm{a}, \mathrm{b}}$, Bin Wen ${ }^{\mathrm{a}}$, Juxiang Zhou ${ }^{\mathrm{b}}$, and Jianhou Gan ${ }^{\mathrm{b}, *}$ \\ ${ }^{a}$ School of Information Science and Technology, Yunnan Normal University, Kunming, 650500, China \\ ${ }^{b}$ Key Laboratory of Educational Informatization for Nationalities of Ministry of Education, Yunnan Normal University, Kunming, 650500, China
}

\begin{abstract}
At present, the sharing and dissemination of cultural resources of minorities in China remain at the early information service stage based on search engine and database query, with slow content updates, closed structure, independence from other databases, and disconnection of content, and they are far from meeting the actual needs of the sharing and dissemination of cultural resources of ethnic minorities. Therefore, aiming at the heterogeneity problem in the sharing and service of minority cultural resources and based on theories and methods such as domain ontology and Map Reduce, this paper first constructs a multi-source heterogeneous integrated model of massive minority cultural resources, Then, an example of Wa nationality is applied to construct the resource domain ontology of ethnic minorities and expand the domain. Finally, on the basis of semantic distance, a method of weighted comprehensive semantic similarity calculation is proposed and verified. The experimental results show that the similarity of the parent node and each child node in Wa hierarchical tree is different, and the similarity result is more reasonable than the original method.
\end{abstract}

Keywords: ontology; semantic distance; ontology integration; minority culture

(Submitted on October 21, 2018; Revised on November 25, 2018; Accepted on December 28, 2018)

(C) 2019 Totem Publisher, Inc. All rights reserved.

\section{Introduction}

In 2017, the General Office of the Central Committee of the Communist Party of China and the General Office of the State Council issued the "Opinions on Implementing the Inheritance and Development of Chinese Excellent Traditional Culture" [1]. On the research of data integration, Zhu et al. proposed a comprehensive event, the ontology similarity calculation method, which has more advantages in event-oriented big data integration [2]. A specific paradigm focusing on semantic data integration, such as Giuseppe De Giacomo's ontology-based data access (OBDA), points to the technologies underlying the paradigm and the major challenges that remain to be addressed [3]. Nguyen et al. proposed a fuzzy ontology integration using the consensus method to solve the conceptual hierarchy of ontology conflicts [4]. Cheng et al. combined semantic similarity with weight salary to conduct semantic mapping and to integrate classification systems of different sources [5]. Wang et al. proposed a knowledge yuan semantic integration method based on fuzzy Petri net [6]. Shi et al. proposed an ontology integration strategy for three kinds of coal mine safety ontology [7]. Li et al. proposed an integrated model based on hybrid ontology [8]. Fang proposed a knowledge fusion method based on key attributes and applied this method to the data integration processing system to improve the rationality of automatic merging of heterogeneous data [9]. Dong proposed a heterogeneous data integration model of university functional departments based on ontology integration from the perspective of ontology engineering [10]. Chen proposed to eliminate the feasibility of the heterogeneous problem among multi-source data by reference data preprocessing and ontology integration [11]. Sang et al. proposed a method of extracting and matching semantic vectors of documents and ontology to solve the problem of knowledge integration [12]. Liu proposed a semantic integration method for oil production engineering data based on domain ontology for the semantic heterogeneity of data in oil production engineering [13]. Li et al. proposed a semantic integration method based on extraction rules and ontology mapping for the well XML (We XML) semantic integration and query application of oil and gas wells [14]. Zheng proposed an automatic semantic retrieval and visualization model based on ontology integration [15]. $\mathrm{Xu}$ et al. proposed a method to calculate the similarity of ontology concepts based on tree structure to solve the complex

* Corresponding author.

E-mail address: ganjh@yunu.edu.cn 
problem of current domain ontology concept similarity [16].

Although domestic and foreign scholars have conducted in-depth research on data integration, few minorities have been involved. Due to the heterogeneity, dispersion, and closure of these ethnic resources, the cognition of ethnic cultural resources is rare in the outside world. Moreover, due to its unstructured characteristics, such knowledge is more difficult to be discovered and applied, let alone the so-called "integration". Therefore, this paper aims at the characteristics of minority cultural resources and the existing heterogeneity problems. Under the MapReduce framework, we construct a multi-source heterogeneous model of minority cultural resources integration at the outset, which presents the transparency, consistency, sharing, interactivity, implement sharing, and reuse of multi-source heterogeneous minority cultural resources. Then, a new method of weighted comprehensive semantic similarity calculation based on semantic distance is proposed. The experimental results show that the similarity between the parent node and each child node in the resource ontology tree of minorities is different.

\section{Related Concepts and Theories}

\subsection{Ontology Concept}

Neches et al. defined ontology as "giving the basic terms and relationships that make up the vocabulary in the relevant field, and the rules that make use of these terms and relationships." At present, the wider accepted-definition is that ontology is a clear formal specification of the shared conceptual model [17]. In heterogeneous data integration, ontology can provide a unified conceptual interface for heterogeneous data sources. The role of ontology is mainly to conduct common semantic descriptions, query models, and reasoning bases [18].

\subsection{Ontology Integration Method}

In terms of ontology data integration, ontology is mainly used to describe the semantics of data sources. Nowadays, there are three main methods of ontology integration: single ontology integration, multi-ontology integration, and hybrid ontology integration. The single ontology approach connects all data sources to a global ontology. For the multi-ontology approach, each data source has an ontology to describe. The hybrid ontology integration approach has a shared vocabulary. In addition, the data sources are described by their respective ontology. A detailed comparison of the ontology integration approach is shown in Table 1. This paper improves the hybrid ontology integration method under the MapReduce framework to complete the integration of minority cultural resources.

Table 1. Comparison of ontology integration methods

\begin{tabular}{|c|c|c|}
\hline \multicolumn{3}{|c|}{ Comparison of ontology integration methods } \\
\hline The way & Advantage & Disadvantage \\
\hline Single ontology method & The structure is relatively simple and easy to implement & It's not easy to add new data sources \\
\hline Multi-ontology method & $\begin{array}{l}\text { There is no absolute correlation between the local ontology and the data source, } \\
\text { making it easier to operate on the data source }\end{array}$ & $\begin{array}{l}\text { Difficult to compare between } \\
\text { different data sources }\end{array}$ \\
\hline Hybrid ontology method & $\begin{array}{c}\text { Data source updates are easier, and mapping rules and shared vocabularies do not } \\
\text { need to be changed to support ontology evolution }\end{array}$ & Insufficient integration scalability \\
\hline
\end{tabular}

\section{Construction of Integrated Model of Minority Culture Information Resources}

\subsection{Integration Model of Heterogeneous Massive Minority Cultural Resources}

In order to solve the heterogeneous problem in the integration of massive ethnic cultural resources, the ontology is introduced as a tool into the integration model. According to the MapReduce framework, the hybrid ontology integration method combining global ontology and local ontology is used to integrate minority cultural resources. The sharing and reuse of minority cultural resources are also realized.

The multi-source heterogeneous massive cultural resource integration model of ethnic minorities is mainly composed of three layers: application layer, middle layer, and data layer. The data layer is located at the bottom of the model. It is formed by structured, semi-structured, and unstructured minority cultural resources. The middle layer is mainly comprised of the mapping rule base, ontology base of minority cultural resources, and query module. The MapReduce method is combined with the hybrid ontology integration algorithm to perform unified data structure and integrated processing on the underlying minority resources. The application layer is mainly for implementing interactions with users, accepting user query requirements, and feeding query results to users. The model is shown in Figure 1. 


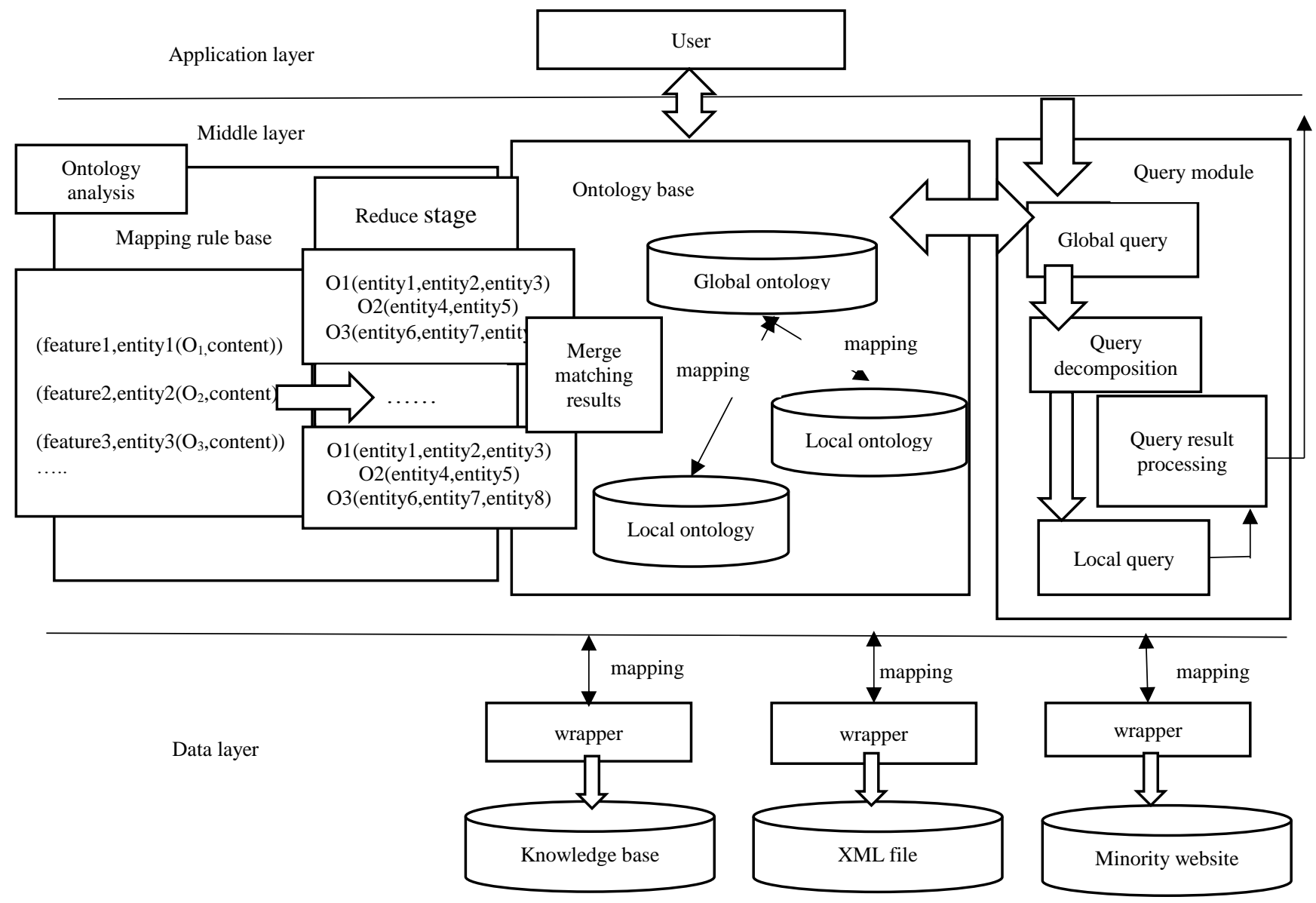

Figure 1. Integration model of minority cultural resources

\subsubsection{Data Layer}

The data layer is the bottom layer of the model and stores data. This layer mainly includes the data source and the wrapper. The data source is partly derived from some existing high-quality knowledge bases, Freebase, YAGO, Excel tables, semistructured XML files, HTML files, and unstructured data storage systems such as minority websites. Wrappers are principally used to encapsulate Wa cultural resources. According to the mapping relationship between the local ontology and the data source, the sub-query is used. A subquery is translated into a query language relating to the data source, and then the wrapper feeds the results back to the middle tier in the XML language (where heterogeneous Wa resources are translated into a uniform data format).

\subsubsection{Middle Layer}

The middle layer is the most important part of the whole model. It consists of three parts: the mapping rule base, the library of minority cultural resources, and the query module. The mapping rule base introduces the MapReduce framework when calculating the similarity in the minority ontology mapping. The first step is parsing the ontology, which is processed in the Map phase. The second step of the parallel scheme is to divide the generated key-value pairs so entities with the same Feature are aggregated onto the same Reduce. The third step of parallelization is to complete the similarity calculation. Since the scheme puts the feature as a key, entities with the same characteristics are divided into the same Reduce. Then, each Reduce performs secondary clustering on the received entity information according to the ontology identifier O. Next, the entity similarity is calculated between each pair of ontology identifiers, and the specific similarity calculation method is described in detail below. The output of different Reduces may have the same similarity result. Therefore, the final output of each Reduce needs to be merged for the final result, as shown in Figure 1. The application layer is user-oriented. The query module will obtain the global query information entered by the user through the application layer. Then, according to the relevant conceptual information in the global ontology, the global information will be instantiated into a unified global query statement and decomposed into several sub-data source query information. Finally, the local query or subquery results 
are integrated and displayed to the user.

\subsubsection{Application Layer}

The application layer is sited at the top of the model and is mainly composed of an application and a browser client. The application layer provides a unified query interface for the user. Users can perform corresponding query operations on minority cultural resources through the application interface layer. It is unnecessary for users to consider the underlying integrated data, which brings convenience and humanization to education businesses, researchers, and ethnic cultural managers. It plays a positive role in promoting the full development, utilization, and dissemination of ethnic minority culture, inheritance and protection of ethnic culture, development of local economy of ethnic minorities, and promotion of cultural exchanges among multi-ethnic groups. Meanwhile, it also lays a foundation for both the management and personalized service recommendation of ethnic minority resources.

\subsection{Constructing the Core Domain Ontology of Minority Resources}

This paper deals with the cultural resources of minorities and takes the heterogeneous cultural resources of Wa nationality as an example to extract the concept and relationship, so as to obtain a set of concepts and relations related to the cultural resources of ethnic minorities.

The construction of Wa core domain ontology involves national history, culture, clothing, and other aspects. Due to the particularity of the national domain itself, the research results of the predecessors are few. There are few core entities in the minority domain on the Internet, which cannot be reused. Thus, it is necessary to collect the related cultural resources of Wa nationality and then sort out the concept of Wa cultural resources. The concept, using natural language processing, extracts the relationship between two groups of concept and forms a concept triplet file.

Wa, for example, using protégé4.3 to construct the core domain ontology. The Wa domain ontology mainly contains seven concept sets including clothing, traditional festivals, customs and habits, social profile, social geography, historical process, and economy. See Figure 2 for details.

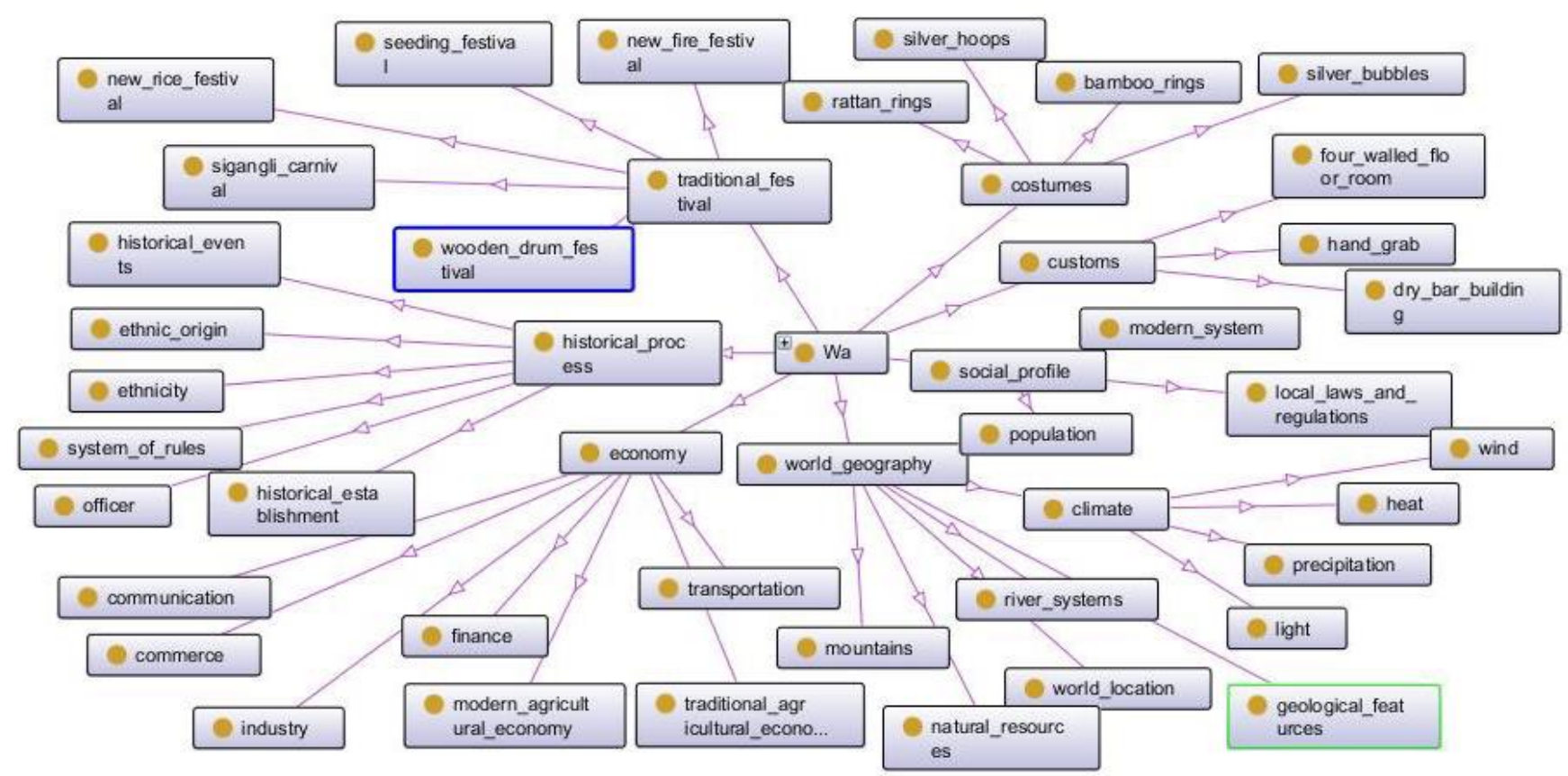

Figure 2. Wa domain ontology

Using the Java development toolkit Jena3.5.0 to implement ontology parsing, the package provided by Jena can parse and manipulate the OWL ontology files in the Wa cultural resource domain. For example, add the sub-concept under the parent concept through the provided interface, parse out the ontology tree classes, properties, class description values, etc., and store in the MySQL database. The results of the ontology analysis are shown in Figure 3. 


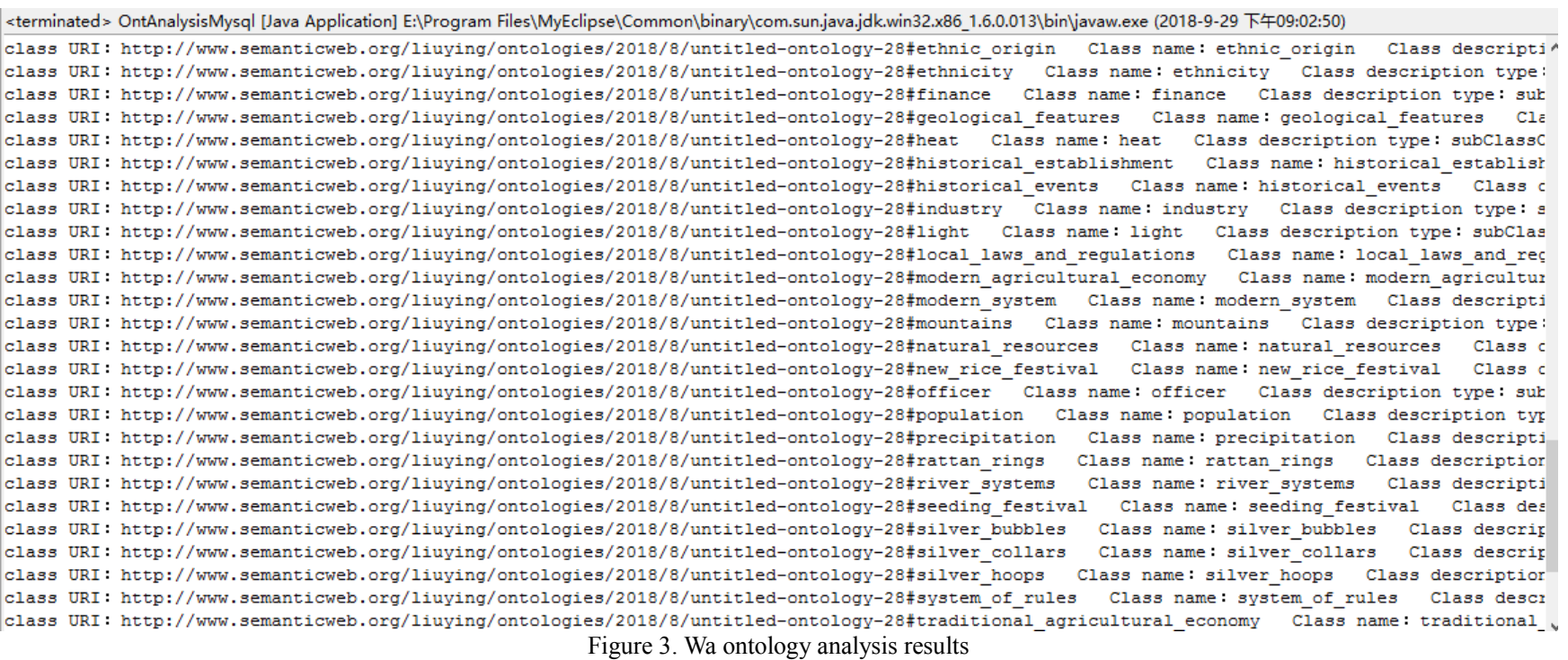

\section{Improvement Algorithm of Domain Comprehensive Similarity based on Semantic Distance}

The key step in ontology mapping is similarity calculation. The semantic distance, information content, attributes, and mixed semantic similarity calculation are mixed in the traditional semantic similarity calculation [19]. In this paper, ontology structure factors (depth, density, width) and semantic distance weighting are integrated to complete semantic similarity calculation of ontology concepts.

\subsection{Concept Width Factor}

The concept width refers to the number of direct child nodes owned by the concept in the ontology hierarchy tree. In the ontology of minority cultural resources domain, the direct sub-nodes corresponding to different concepts are also different. A larger width indicates that the concept tends to be more detailed. Without considering other factors, the wider the concept, the smaller the similarity between the concept child nodes. By adding the influence of concept width on semantic similarity calculation, the semantic similarity of two concepts $X$ and $Y$ in concept width can be obtained. This is shown in Equation (1), where $\omega$ and $\varphi$ are the adjustment parameters of conceptual width.

$$
\operatorname{Sim}_{\text {wid }}(X, Y)=\sqrt{\frac{\omega}{\operatorname{wid}(X)+\omega}} \times \sqrt{\frac{\varphi}{\operatorname{wid}(Y)+\varphi}}
$$

\subsection{Concept Depth Factor}

The ontology conceptual model is a hierarchical tree. The top-down concept is increasingly more concrete. When other factors are not considered, the semantic similarities of the two concepts $X$ and $Y$ in the upper layer are obviously better than those in the lower one. The similarity of concepts is small, so we introduce the depth in the hierarchical tree into the calculation of semantic similarity. The specific similarity calculation measure is shown in Equation (2).

$$
\operatorname{Sim}_{\text {dep }}(X, Y)=\frac{1}{2}\left(\frac{\operatorname{dep}(X)+\operatorname{dep}(Y)}{\mid \operatorname{dep}(X)-\operatorname{dep}(Y)+2 \operatorname{dep}(\text { Tree }) \mid}+\frac{\operatorname{dep}(\text { LCA })}{\operatorname{dep}(\text { Tree })}\right)
$$

LCA (minimum common ancestor node depth) node depth is introduced in the calculation of conceptual depth similarity and solves the unreasonableness of the original algorithm. When the depth of the hierarchical tree is deep enough, the similarity of two leaf nodes in the root node is quite large, which does not conform to the actual situation [20].

\subsection{Concept Density Factor}

Concept density is the proportion of a subtree whose root is a concept node in an ontology hierarchical tree. The density of 
conceptual nodes at different levels of concept hierarchy in the concept hierarchy tree will also be different. The deeper the subtree of the concept node in the concept hierarchy tree, the denser the representation here, the more detailed the concept as well, and the less semantic information each child node undertakes. The similarity between the sub-concept pairs is smaller. The node concept similarity calculation measure is shown in Equation (3).

$$
\operatorname{Sim}_{\text {den }}(X, Y)=\frac{\operatorname{dep}(\text { Tree } X)}{\operatorname{dep}(\text { Tree })} \sqrt{\frac{\operatorname{Count}(\text { TreeX })}{\operatorname{Count}(\text { Tree })}} \times \frac{\operatorname{dep}(\text { TreeY })}{\operatorname{dep}(\text { Tree })} \sqrt{\frac{\text { Count }(\text { TreeY })}{\text { Count }(\text { Tree })}}
$$

Where $\operatorname{dep}($ Tree $X)$ represents the depth of the concept $X$ subtree, Count(TreeX) represents the number of child nodes rooted at the concept $X$, and Count(Tree) represents the total number of nodes in the ontology hierarchy tree.

\subsection{Semantic Distance Factor}

In the concept hierarchy tree, the semantic distance is usually expressed by the shortest path length between two concepts. When the two concepts $X$ and $Y$ have a semantic distance of 0 , the similarity is 1 . When the semantic distance between the two concepts $X$ and $Y$ is infinity, the semantic similarity is 0 , that is, the narrower the semantic distance between two concepts, the greater the semantic similarity. The semantic distance is inversely proportional to the semantic similarity, and the semantic distance similarity calculation measure is shown in Equation (4).

$$
\operatorname{Sim}_{\text {dist }}(X, Y)=\frac{2(\text { length }-1)-\operatorname{dis}(X, Y)}{2(\text { length }-1)}
$$

\subsection{Improved Comprehensive Similarity Algorithm (SDA) based on Semantic Distance}

Based on the semantic distance-based weighted synthetic semantic similarity algorithm, the ontology structure (width, density, and depth) factors and the semantic distance between ontology are introduced in the similarity calculation of the minority cultural resource ontology concept. The weighted similarity is calculated by Equation (5), which is from Equations (1) to (4).

$$
\operatorname{Sim}(X, Y)=\alpha \operatorname{Sim}_{w i d}(X, Y)+\beta \operatorname{Sim}_{d e p}(X, Y)+\gamma \operatorname{Sim}_{\text {den }}(X, Y)+\delta \operatorname{Sim}_{\text {dist }}(X, Y)
$$

Where $\alpha$ is the width factor, $\beta$ is the depth impact factor between the conceptual levels, $\gamma$ is the conceptual density impact factor, $\delta$ is the semantic distance impact factor, and $\alpha+\beta+\gamma+\delta=1$.

\section{Experiment Results and Analysis}

The conceptual hierarchy diagram of the cultural resource ontology of minorities is shown in Figure 4. Set the depth of the root of Thing to 1 and the weight of the directed edge between the concept and the concept to 1. Select the ethnic costumes, ethnic diets, and ethnic customs in the figure, and calculate the semantic similarity between ethnic costumes and ethnic diets, ethnic diets and ethnic customs, and ethnic customs and ethnic costumes respectively according to the improved algorithm, as shown in Table 2 .

Table 2. Comparison before and after semantic similarity improvement based on semantic distance

\begin{tabular}{|c|c|c|c|}
\hline \multicolumn{1}{|c}{ Table 2. Comparison before and after semantic similarity improvement based on semantic distance } \\
\hline Algorithms & Ethnic costumes and Ethnic diet & Ethnic diet and ethnic customs & Ethnic customs and national costumes \\
\hline Semantic distance algorithm & 0.667 & 0.833 & 0.833 \\
\hline SDA algorithm & 0.576 & 0.644 & 0.642 \\
\hline
\end{tabular}

The value of the adjustment parameter $\omega, \varphi$ are set by the domain expert [21]. After a number of tests of $\alpha, \beta, \gamma, \delta$, the following two sets of impact factors are the most suitable.

$$
\mathrm{A}: \alpha=0.1, \beta=0.2, \gamma=0.1, \delta=0.6, \mathrm{~B}: \alpha=0.15, \beta=0.15, \gamma=0.1, \delta=0.6
$$

As can be seen from Table 2, ethnic diet and national customs are considered only when the semantic distance is 
considered for similarity calculation. The similarity of national customs and national costumes is consistent, both of which are 0.833 . After the improvement, the similarities of ethnic diet and ethnic customs as well as ethnic customs and ethnic groups are 0.644 and 0.642 respectively. It can be seen that the similarity between the parent node and the different child nodes is different, and the similarity between ethnic diet and ethnic customs is higher.

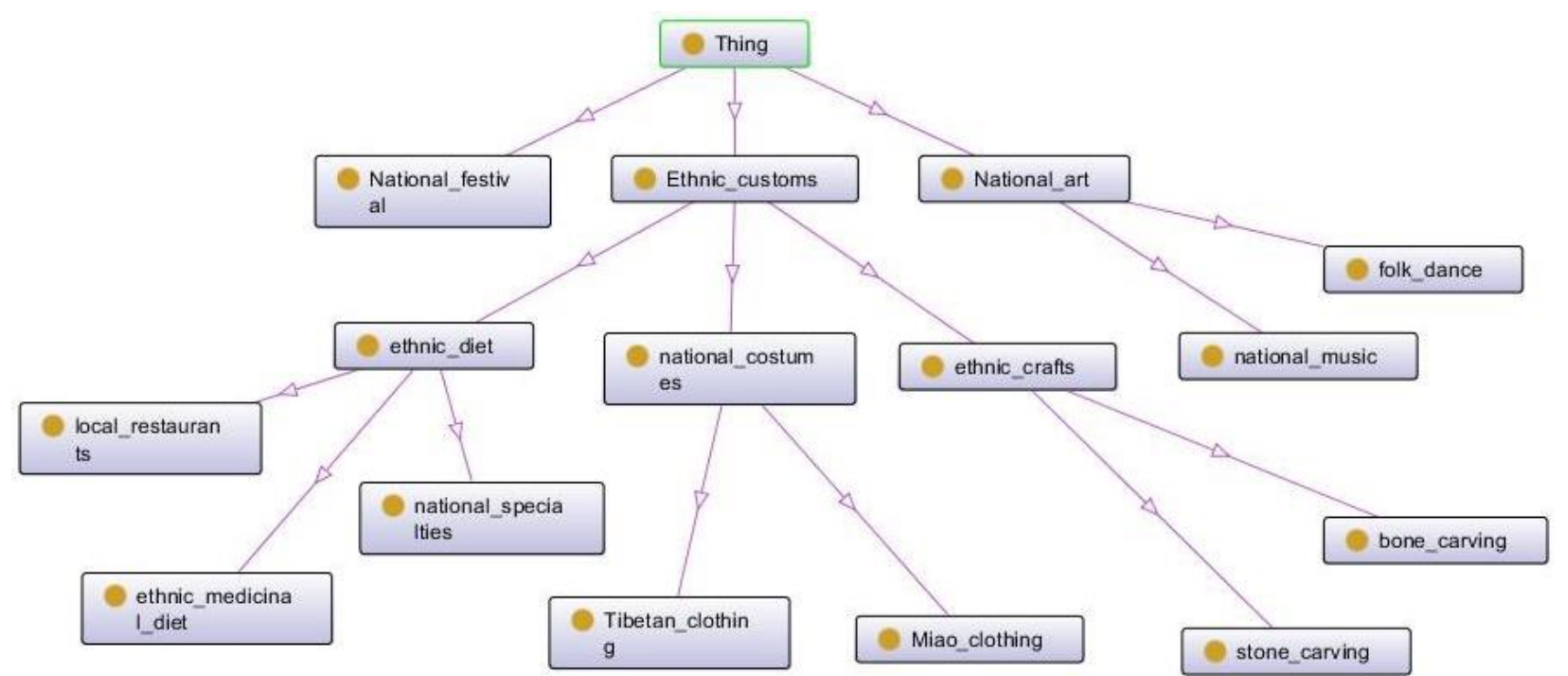

Figure 4. Conceptual hierarchy diagram of part of the cultural resource ontology of minorities

It can be seen from Table 3 that when the density factor and semantic distance factor are the same and the width factor is 0.15 , the similarity between ethnic costumes and ethnic diets, ethnic diets and ethnic customs, and ethnic customs and ethnic costumes are $0.563,0.640$, and 0.639 respectively. When the width factor is 0.1 , the width is inversely proportional to the semantic similarity. The depth is inversely proportional to the semantic distance in the same way.

Table 3. Semantic similarity comparison under different weight values

\begin{tabular}{|c|c|c|c|}
\hline Weights Concepts & National costumes and national diet & Ethnic diet and ethnic customs & National customs and national costumes \\
\hline A & 0.576 & 0.644 & 0.642 \\
\hline B & 0.563 & 0.640 & 0.639 \\
\hline
\end{tabular}

\section{Conclusions}

For the purpose of solving the heterogeneity and dispersion of minority cultural resources, a multi-source heterogeneous cultural resource integration model is constructed at the beginning. Then, the domain of the Wa community is constructed and the analytical extension is performed. In the end, an improved method for calculating the synthetical semantic similarity is proposed based on the semantic distance, combining the ontological structure factors (width, depth, density) and semantic distance. Experiment results show that the similarity between parent and different child nodes is different in the minority ontology hierarchical tree. The improved semantic similarity calculation method improves the accuracy of domain ontology similarity. It is closer to the real semantic similarity, more in line with the experience of domain experts, and avoids the uniqueness and one-sidedness of the computing process, which lays a solid foundation for the mapping between ontologies and the subsequent query expansion. However, there are still some improvements in the semantic similarity calculation. The attribute factor will be taken into account in future works, and the algorithm will be further processed under the Map Reduce framework.

\section{Acknowledgments}

This research is supported by the National Nature Science Fund Project (No. 61562093, 61661051), Key Project of Applied Basic Research Program of Yunnan Province (No. 2016FA024), Program for Innovative Research Team (in Science and Technology) in University of Yunnan Province, Application Infrastructure Projects of Science and Technology Plan in Yunnan Province (No. 2016FD022), and Starting Foundation for Doctoral Research of Yunnan Normal University (No. 2017ZB013). 


\section{References}

1. X. K. Chen, "An Analysis of the Historical Development of The Inheritance and Protection Policy of New Chinese National Culture," Studies of Ethnic Higher Education, Vol. 5, No. 3, pp. 27-33, July 2017

2. W. Y. Zhu, W. Liu, and Z. T. Liu, "Comprehensive Approach for Event Ontology Similarity Computation," Journal of Computer Applications, Vol. 36, No. 8, pp. 1-3, April 2018

3. G. D. Giacomo, D. Lembo, and M. Lenzerini, "Using Ontologies for Semantic Data Integration," A Comprehensive Guide Through the Italian Database Research Over the Last 25 Years, Vol. 5, No. 31, pp. 187-202, May 2017

4. N. T. Nguyen, B. Trawiński, and J. J. Jung, "New Challenges for Intelligent Information and Database Systems," Studies in Computational Intelligence, January 2011

5. Z. B. Cheng, T. Y. Shi, and Y. J. Wang, "Semantic Integration Method of Railway Geographic Information Classification based on Formal Ontology," Railway Transport and Economy, Vol. 39, No. 1, pp. 88-94, March 2017

6. J. Wang, C. S. Liu, and C. X. Qin, "Semantic Integration Method of Knowledge Elements based on Fuzzy Petri Net," Information Studies Theory \& Practice, Vol. 40, No. 9, pp. 140-144, October 2017

7. X. L. Liu, X. H. Liu, Q. P. Shi, et al., "Research on Coal Mine Safety Ontology," Industrial and Mining Automation, Vol. 44, No. 3, pp. 42-49, March 2018

8. Y. Z. Li, et al., "Research on E-Government Heterogeneous Data Integration with Hybrid Ontology Method," Journal of University of Electronic Science and Technology of China (Social Sciences Edition), Vol. 18, No. 5, pp. 17-20, October 2016

9. L. F. Fang, "Ontology-based Heterogeneous Data Integration and Fusion Method," University of Science and Technology of China, May 2010

10. C. Dong, "Research on Information Isolated Islands in Universities' Functional Department based on Ontology Integration," Central China Normal University, May 2015

11. Y. P. Chen, "Ontology-based Vector Data Consistency Check," Zhejiang University, May 2017

12. J. Cheng, C. Sang, and Y. M. Shi, "Knowledge Integration and Semantic Annotation in Closed-Loop Lifecycle Management System," Journal of Computer Applications, Vol. 37, No. 6, pp. 1728-1734, July 2017

13. X. Liu, "Research on Key Technologies of Domain Data Integration and Service," University of Science and Technology Beijing, December 2016

14. H. Y. Li, H. Xiao, and P. Y. Zhang, "Domain XML Semantic Integration based on Extraction Rules and Ontology Mapping," Journal of Hebei University of Science and Technology, Vol. 37, No. 4, pp. 416-422, August 2016

15. W. Q. Zheng, “Automatic Semantic Retrieval and Visualization Model based on Ontology Integration," Information Science, Vol. 31, No. 5, pp. 77-83, May 2013

16. H. Jia and Y. Z. Xu, "Ontology Concept Similarity Calculation based on Tree Structure," Journal of Computer Systems, Vol. 26, No. 3, pp. 275-279, March 2017

17. H. Z. Li, X. Q. Wang, and B. L. Zhang, "Overview of Ontology Research,” Journal of Intelligence, Vol. 35, No. 6, pp. 163-170, July 2016

18. J. Y. Pan, "Research on Ontology-based Heterogeneous Data Integration," Donghua University, December 2013

19. H. X. Sun, et al., "A Review of Research on Semantic Similarity Calculation Methods based on Ontology," New Library and Information Technology, Vol. 26, No. 1, pp. 51-56, January 2010

20. H. Zhang, et al., "Improved Ontology-based Semantic Similarity Calculation Algorithm," Computer Engineering and Design, Vol. 36, No. 8, pp. 2206-2210, September 2015

21. Y. X. He, B. M. Shi, and Y. Zhang, "Research on Ontology-based Semantic Similarity Algorithm," Computer Applications and Software, Vol. 30, No. 11, pp. 312-315, December 2013

Ying Liu is a Master's student in the School of Information Science and Technology at Yunnan Normal University. Her research interests include natural language processing and semantic web.

Bin Wen received his Ph.D. in computer application technology from China University of Mining \& Technology in 2013. Currently, he is an associate professor at Yunnan Normal University. His research interests cover intelligent information processing and emergency management.

Juxiang Zhou received her Master's degree in 2011 from Yunnan Normal University. Currently, she is an assistant research fellow at Yunnan Normal University and is pursuing a Ph.D. at Dalian University of Technology. Her research interests include image processing and pattern recognition.

Jianhou Gan received his Ph.D. in metallurgical physical chemistry from Kunming University of Science and Technology in 2016. Currently, he is a professor at Yunnan Normal University. His research interests cover education informatization for nationalities, semantic web, databases, and intelligent information processing. 